For Routledge Handbook of Philosophy of Physics (Eds. E. Knox and A. Wilson)

\title{
Non-causal explanations in physics
}

\section{Juha Saatsi}

School of Philosophy, Religion, and History of Science

Woodhouse Lane, Leeds LS2 9JT

University of Leeds

J.T.Saatsi@leeds.ac.uk

Juha Saatsi is Associate Professor at University of Leeds. He works on various topics in philosophy of science, and he has particular interests in the philosophy of explanation and the scientific realism debate.

\section{Introduction}

The nature of scientific explanation and understanding are of central interest to philosophers of science. How (or by virtue of what) does a given theory, model, or body of information explain? What is the relationship between what is being explained (the explanandum), and what is doing the explaining (the explanans)? These questions lie at the heart of general philosophy of science, where they connect in important ways to broader issues concerning, e.g., scientific realism and the nature of scientific inferences, evidence, and theory-choice (see Saatsi 2017a for a recent review). Over the last decade or so these debates have become increasingly deeply informed by the history and philosophy of physics.

Since the demise of the Deductive-Nomological ('DN') account of explanation in the 1970s and 80 s, philosophy of explanation has been largely dominated by the idea that explanation is intimately associated with causation. Unificationist theories of explanation have been the only serious competitor to causal-mechanical accounts for much of the period spanning from Hempel and Oppenheim's 'epoch-making' DN-account (1948) to the current state of the art (see Salmon 1989 for a classic review). Some of the best developed and most influential contemporary accounts, e.g. by Woodward (2003) and Strevens (2008), have further reinforced the hegemony of causal theories of explanation (the latter putting a unificationist spin on the causal ideology). 
For Routledge Handbook of Philosophy of Physics (Eds. E. Knox and A. Wilson)

Undoubtedly there are countless explanations in physics that nicely fit the intuitive causal ideology, according to which explanatory information is suitably regimented causalmechanical information (see the chapters by Laura Felline and Mattahias Frisch in this volume). But looking at the explanatory practices of physics more broadly, it is also easy to identify numerous examples of seemingly non-causal explanations. Detailed studies of such explanations have led to steadily growing interest in understanding exactly how these explanations work: how they explain, and how they differ from causal explanations. There are also substantial issues concerning the relationship between causal and non-causal explanations, and whether some phenomena can only be explained in non-causal terms. I will review some prominent answers to these questions, but first I will bring out the richness of our subject matter with a menagerie of explanations that have been regarded as non-causal.

\section{A menagerie of non-causal (?) explanations}

I have divided the examples below under three headings, involving (i) geometry, (ii) symmetries, and (iii) inter-theoretic relations. These broad headings serve to regiment the discussion for presentational purpose first and foremost, and many of these explanations cut across this 'classification' in interesting ways. For the sake of brevity, I will only sketch each case; fuller details can be found in the literature cited. Whether all of these explanations really are non-causal is itself a contested issue to be discussed in the next section.

Explanations involving geometry. Inertially moving particles in curved space can (say) decrease in distance over time due to the 'shape of space'. Since no forces are involvedinertial particles are moving straight along geodesics — one may want to say that nothing causes this change in the particles' relative distance. Rather, what explains it is the curvature of space. This exemplifies a simple geometrical explanation according to Nerlich (1979).

Geometrical explanations can also involve global features of space, such as its dimensionality, or orientability. Asymmetrical objects, like hands, can be enantiomorphic if they are embedded in orientable space of appropriate dimensionality. No continuous movement in two-dimensional orientable space takes a left-handed rigid L-shape to match a corresponding right-handed one, for instance. Add a third dimension, or make your twodimensional space non-orientable (e.g. Moebius strip), then such continuous transformations do exist. What determines - and thus arguably explains, in some sense-that two objects are 
For Routledge Handbook of Philosophy of Physics (Eds. E. Knox and A. Wilson)

enantiomorphic (or otherwise), are global features of space in which these objects are 'embedded' (Nerlich 1979).

In a somewhat similar manner, it has been suggested that in Newtonian mechanics an explanatory relationship holds between the dimensionality of space, on the one hand, and the stability of planetary orbits, on the other. Although there is disagreement here which way the explanation really runs - this can depend, e.g., on whether we are relationists or substantivalists about Newtonian space (see Callender 2005) - the basic idea turns on the fact that natural generalisations of Newtonian gravitational potential to $n$-dimensional $(n>3)$ spaces implies the impossibility of stable periodical gravitational orbits (Ehrenfest 1917, Buchel 1969). If we regard the dimensionality of space as a specific global feature of the world that could have been different, whilst holding the rest of Newtonian dynamics fixed (by generalising Newtonian gravitational potential in a natural way), then the stability of planetary orbits depends on - and thus is arguably explained by, in some sense - this global feature of the space in which the planets orbit (see e.g. Woodward 2018).

The examples above involve features of physical space. Physical theories of course deal also with various kinds of more abstract spaces, the geometrical features of which can be explanatorily relevant as well. Kinematics (in contrast to dynamics) is sometimes characterised as 'the study of the geometry of motion' - the study of the geometrical features of physical systems' possible configurations, relating e.g. displacement, velocity, acceleration, and time, without reference to forces or causes of motion. Some purely kinematic explanations are naturally contrasted with causal explanations.

For a paradigmatic toy example, consider the space of possible instantaneous configurations of a stick the origin of which is confined to a curved 2-dimensional surface: think of spherical surface, say, and its tangent vectors. Some of these states are related to one another by parallel transport, viz. moving the stick along the surface so that it is kept 'straight' along the way. Now, why does the stick, having been parallel-transported over a closed loop on a curved surface, return to the starting point so that its direction differs by angle $\alpha$ from the initial configuration? The standard geometrical explanation turns on the way in which $\alpha$ is determined by — and thus arguably explained by, in some sense - the curvature of the surface and the area enclosed by the path. (For spherical surface, this is simply given by the equation $\alpha=\mathrm{r}^{-2} \times \mathrm{A}$, where $\mathrm{A}$ is the enclosed area and $\mathrm{r}$ is the radius.) These non-local geometrical 
For Routledge Handbook of Philosophy of Physics (Eds. E. Knox and A. Wilson)

features do not seem causally responsible for $\alpha$, and none of the dynamical features or laws of nature (governing how the stick moves around in the specified manner) feature in the explanation either, making this a plausible example of non-causal explanation (Saatsi 2018).

The ideology of this paradigmatic kinematic explanation arguably also applies to broadranging phenomena of 'geometrical phase' of suitable dynamical systems, involving, e.g., the precession of Foucault's pendulum and other similar exemplifications of the so-called Hannay's angle that shows up when a classical dynamical system travels a closed loop in a parameter space without returning to its original state. (The daily precession $\alpha^{\prime}$ experienced by a Foucault pendulum, as a function of the latitude $\lambda$, is given by $\alpha^{\prime}=-2 \pi \sin \lambda$. For a geometrical explanation see e.g. von Bergmann and von Bergmann 2007.) It also generalises to various other areas of physics where a system's parameter space has a rich enough structure to give rise to geometrical (an)holonomies upon which some explanandum phenomenon can depend. Interesting examples can be found in, e.g., classical optics, nuclear magnetic resonance, hydrodynamics, and quantum field theory (see Wilczek et al. 1989). The much-studied phenomenon of Berry phase in quantum mechanics is a particularly important and well-known example (see Batterman 2003, Lyre 2014, Saatsi 2018).

The space of a physical system's possible configurations can also have global topological features that can be deemed to be explanatory in a non-causal way. For example, why do all double pendulums have at least four equilibrium configurations? Arguably a critical part of this explanation turns on the fact their configuration space is a torus - a fact that holds regardless of the specifics of pendulum design and dynamics, and even regardless of the specifics of the dynamical laws that connect force to acceleration (Lange 2017). This is a very simple example of a topological explanation, well suited for philosophical discussion and analysis; more involved real-life explanations involving topological notions abound, ranging from the Aharonov-Bohm effect to topological phases of matter. (See Healey 2007 for in-depth philosophical discussion of the Aharonov-Bohm effect. Little has been written, in general, about topological explanations in physics.)

Explanations involving symmetries. Amongst the numerous and varied examples of symmetry-based explanations in physics one finds appeal to both discrete and continuous symmetries. I will give a paradigmatic example of each. 
For Routledge Handbook of Philosophy of Physics (Eds. E. Knox and A. Wilson)

In many-particle quantum mechanics a system's wavefunction must be either symmetric or anti-symmetric under particle permutations. This exemplifies a discrete symmetry. This fundamental symmetry underlies quantum statistics, and it plays an indispensable explanatory role in explanations of various physical phenomena. For instance, the Pauli Exclusion Principle (PEP) for fermions follows from this symmetry, dictating that no two electrons (say) can be in the same quantum state. Various explanations involving PEP - including, e.g., the stability of matter, various features of chemical bonding, and the halting of white dwarf collapse - can be viewed as non-causal explanations: these phenomena are arguably essentially explained, not by reference to any causal features of the world, but rather in terms of a fundamental global kinematic constraint on the possible physical states of multi-particle quantum system (see French and Saatsi 2018, Skow 2014).

For a specific example, consider the explanation of solubility of salt, for instance. An important part of the explanation turns on the role of the so-called 'Pauli repulsion' in determining the chemical bond-dissociation energy for the ionic bond between $\mathrm{Na}+$ and $\mathrm{Cl}$ in $\mathrm{NaCl}$ molecules. Despite its name, 'Pauli repulsion' arguably really should not be thought of as involving a force of any kind, or even having a causal basis more broadly construed. Rather, it is grounded in PEP (and the more fundamental quantum symmetry that underlies PEP, ultimately deriving from the so-called permutation invariance of quantum mechanics). In a similar way, the so-called 'degeneracy pressure', which halts the gravitational collapse of a white dwarf star, is arguably best understood in non-causal terms as a kinematic consequence of the same quantum symmetry (see French and Saatsi 2018 for details.).

Moving on to explanatory use of continuous symmetries in physics, consider the intimate connection that Noether's theorem brings out as holding between a system's conserved physical quantities, on the one hand, and its continuous symmetries, on the other. Assuming that we are operating in the framework of Euler-Lagrange equations of motion for fields or particles, the generality of Noether's theorem is remarkable, applying to any symmetry of the system's Lagrangian, whether these involve external degrees of freedom (e.g. homogeneity and isotropy of space), or internal degrees of freedom (e.g. gauge symmetries).

Given the connection brought out by Noether's theorem, we know that if a particular physical system displays some conserved quantities, there is a corresponding symmetry, and vice versa. Can one explain the other? Arguably so: a system's symmetries are often naturally 
For Routledge Handbook of Philosophy of Physics (Eds. E. Knox and A. Wilson)

viewed as determining — and hence, in some sense, explaining — those aspects of the system's behaviour that we identify as the conserved quantities.

For a simple concrete example, consider a classical particle moving under a constant central force. (Its potential energy depends only on the radial coordinate.) Why is the particle's trajectory constrained to a plane? This regularity of the system's dynamics follows from the symmetries of the particle's kinetic and potential energy functions (which together give the Lagrangian, of course). Arguably these symmetries explain the system's constant of motion, because Noether's theorem tells how the latter would have been different, if e.g. the potential energy function were different in some way. For instance, it tells us what the constants of motion would have been, had the potential energy function been time-dependent in a particular way, say.

In the physics literature Noether's theorem is often also associated with the idea that conservation laws can be explained terms of global symmetries. This connection is harder to make sense of without introducing substantial assumptions regarding the metaphysics of laws. Lange (2007) argues that Noether's theorem is altogether irrelevant for accounting for conservation laws, and that symmetries can explain conservation laws only if they are modally stronger 'meta-laws' that govern ordinary laws.

Explanations involving inter-theoretic relations. Various explanatory practices in physics involve relating one theory to another in some systematic, understanding-inducing way. (We are interpreting 'theory' broadly here, to include mathematical models.) The idea of intertheoretic reduction in Nagel's (1961) sense provides an example of this: if two theories are so related that the laws of the reduced theory can be logically deduced from the laws of the reducing theory, given some well-defined bridge-principles that relate the two theories' theoretical vocabularies, then we arguably have a DN-type explanation of the reduced theory by the reducing theory. In these terms we can understand in plausibly non-causal terms, for example, the applicability and effectiveness of the reduced theory as turning on logical connections between two theoretical structures and the synthetic identity claims stated by the bridge-principles.

Insofar as physics features reductions like this (or something close enough), and such reductions really do count as explanatory (see Dizadji-Bahmani et al. 2010), we have found 
For Routledge Handbook of Philosophy of Physics (Eds. E. Knox and A. Wilson)

yet another class of explanations that are plausibly non-causal. Admittedly purely deductive relations between theories in Nagel's spirit are arguably very few and far between - as anyone familiar with the extensive debates on (anti-)reductionism in physics very well knows. (See, however, Dizadji-Bahmani et al. 2010 and Schaffner 2012.) Be that as it may, for our current discussion little hangs on this, since the kinds of inter-theoretic relations capable of supporting non-causal explanations can take many forms other than Nagelian relations of logical deduction. Similarly, whether or not we can uphold any of the various reductionist theses, it is undeniable that much of physics involves attempts to link in explanatory ways theories at different 'levels'. Physics unquestionably offers explanatory understanding aplenty regarding how, e.g., thermodynamic phenomena relates to (both classical and quantum) mechanics; how phenomena described in ray-optics relates to wave optics and electromagnetism; how classical mechanics relates to quantum mechanics; how chemical phenomena relates to quantum mechanics; how phenomena described in Newtonian gravitation relates to general relativity; and so on. The explanatory importance of numerous inter-theoretic relationships uncovered by physics is not eroded by the fact that reductionism faces important challenges in relation to various purported reductions of one theory to another. Furthermore, plausibly many of the explanations furnished by inter-theoretic relations in physics are non-causal, and not amenable to a causal-mechanical (or causal-role functionalist) treatment. I will now briefly sketch two examples.

In Newtonian Gravity (NG) inertial and gravitational mass are conceptually entirely distinct, yet they turn out to be identical. Why? The answer is given, of course, by examining the relevant phenomena in relation to General Relativity (GR). But it is not the case that GR shows how the two concepts of mass get identified: gravitational mass does not feature in GR at all, given that the concept of gravitational force makes no sense in GR. Rather, the explanation involves showing how NG relates to GR, so as to answer the question: "why if GR is true, $[\mathrm{NG}]$ is such an effective theory in some contexts?" (Weatherall 2011, p. 429) Answering this question explains away the seeming coincidence of gravitational and inertial masses, thereby providing an answer to the why-question above. As Weatherall explains: "The explanation consists in showing that if the world is to be understood as a model of GR, then any model of $[\mathrm{NG}]$ that successfully approximates the world, even in limited regimes, must satisfy the conditions that [the two masses are identical]." (p.437) Showing this requires bringing out in detail the relevant relationship between NG and GR, which is done in terms of 
For Routledge Handbook of Philosophy of Physics (Eds. E. Knox and A. Wilson)

showing mathematically how models of (geometrized) NG arise as a limiting case of GR in a rigorous sense of 'flattening' the light-cone structure of GR.

My second example concerns the so-called renormalisation group explanation of critical phenomena that involve (second-order) phase transitions in macroscopic systems near their critical point. Thermodynamic properties near criticality obey power laws as a function of (reduced) temperature. Micro-physically very different systems can obey the same power laws, in which case they belong to the same 'universality class', identified by the exponents of the relevant power laws. These exponents depend only on the system's spatial dimensionality and certain abstract features of the micro-interaction (e.g. the dimensionality of the 'spin' parameter). Why do the macroscopic phenomena quantified by the power lawsobeyed by all systems that belong to the same universality class - only depend on these features?

The answer to this question involves mathematical relationships amongst extremely large classes of possible micro-physical models of statistical physical systems. These relationships are brought out by employing 'renormalization transformations', which form equivalence classes of those models (in the abstract mathematical space of possible models) that share the same long-distance physics. It can be shown that this space-which is assumed to include the models that correctly capture the actual micro-physics of systems that physicists actually measure or simulate - has a rich mathematical structure that reveals the fact that while the power laws are strikingly independent of the specifics of the micro-physical interactions, they only depend on the abstract features mentioned above (Batterman 2002, Saatsi and Reutlinger 2018, Morrison 2015). The explanation furnished by such renormalisation group techniques is again arguably non-causal, turning on rich mathematical relationships in the space of possible models of physical systems of very many interacting parts. (However, see Sullivan, forthcoming, for a criticism of this verdict).

Other examples? Physics features numerous other examples of purportedly non-causal explanations, many of which do not fall neatly under the three broad headings above. For some further examples and 'headings', see, e.g., Lange's (2009) and Pexton's (2014) discussion of dimensional explanations; Lange's (2017, Ch. 5) discussion of 'really statistical'explanations; the discussion of some quantum mechanical explanations in Clifton (1998) and Healey (2017); and Batterman's (2002) discussion of asymptotic explanations. 
For Routledge Handbook of Philosophy of Physics (Eds. E. Knox and A. Wilson)

\section{How do these explanations work?}

How do the above examples of (allegedly) non-causal explanations work? Where do their 'explanatory power' derive from? Philosophers have attempted to articulate the explanatoriness of explanations like these in various ways. While many are happy to do this in non-causal terms, others find ways to regard as causal at least some of the above explanations (e.g., Strevens 2018, Skow 2016). Whether or not a given explanation counts as 'non-causal' will partly depend, of course, how the foil, 'causal explanation', is construed. Even the most ardent current proponents of non-causal explanations regard some of the examples above as causal. For example, Lange (2017) views as causal the simple 'geometrical' explanation involving inertially moving particles in curved space, while he regards as non-causal the explanation involving enantiomorphic figures in an orientable space (see start of §2). According to Lange, the former explanation is causal, "since it works by specifying the causes (forces) that are acting on the dust particles (namely none) as well as the laws and conditions determining how things in the particles' locations would behave were they under the influence of no causes." (p. 403) The latter explanation Lange analyses as a non-causal explanation 'by constraint'. (p. 126)

Given the disagreement about the status of many of the examples cited, one may ask what point there is to demarcate between causal and non-causal explanations? What exactly hangs on it? As I see it, a central aim of philosophy of explanation is to faithfully capture the explanatory practice of science, and to mark significant distinctions that scientists themselves draw between types of explanations. What do physicists regard as explanatory, and why? The contrast between causal vs. non-causal explanations can be a useful way to articulate such distinctions, the philosophical point of such a contrast being underwritten by the corresponding distinctions' significance to science. Scientists do, after all, care what kinds of explanations there are, or could be, and how these relate and contrast with one another (see Lange 2017 and Saatsi 2018).

In the rest of this section I will briefly look at some prominent analyses of non-causal explanations. I do not have space to get into the details of the particular accounts, but I will highlight two competing trends in the currently booming literature. One trend is exemplified by philosophers who emphasise the independence of the explanandum of non-causal explanations from specific causal features of the system in question: many non-causal 
For Routledge Handbook of Philosophy of Physics (Eds. E. Knox and A. Wilson)

explanations have been thought to work by bringing out (in one way or another) such independence. The second, competing trend is exemplified by philosophers who emphasise the (counterfactual) dependence of the explanandum on the explanans, in broad analogy with causal explanations. According to this line of thought many non-causal explanations explain in a way analogous to causal explanations, but by tracking relations of non-causal dependence instead of causal dependence.

Let's begin with the first trend. There are many ways in which many non-causal explanations can be independent of, or abstract away from, causal features. According to Pincock (2007), some non-causal mathematical explanations — such as Euler's well-known graph-theoretic explanation of why one cannot traverse Koenigsberg's bridges in a particular way-work by "abstracting away from the microphysics". And not just microphysics: Euler's explanation is independent of all the physical details regarding the bridges lengths, angles, and so.

According to Batterman and Rice (2014), some scientific models are capable of explaining "universal patterns" amongst micro-physically heterogeneous systems by demonstrating that the micro-details, distinguishing all these systems (as well as the explanatory model system) from one another, are irrelevant for the universal behaviour being explained. These explanatory 'minimal models' are extreme caricatures of real system, but according to Batterman and Rice they do not explain by virtue of capturing any 'common features' between the different systems as explanatorily underlying the universal behaviour. Rather, minimal model explanations arguably work by showing how micro-physically heterogeneous systems can partake in a "universal pattern"- belong to one and the same "universality class", such as those involved in renormalization group explanations-by virtue of some features of the phenomenon at stake that renders irrelevant the details that distinguish the model system and various real systems.

Lange (2017) offers a very different account of non-causal explanations that also in a way exemplifies the trend that emphasises the independence of the explanandum from specific causal features. According to Lange, a broad range of non-causal explanations work "by describing how the explanandum arises from certain facts ('constraints') possessing some variety of necessity stronger than ordinary laws of nature possess" (p.10). He presents varied examples of such 'explanations by constraint', which also include some of the examples discussed above (\$2), such as the topological double-pendulum explanation. Many other 
For Routledge Handbook of Philosophy of Physics (Eds. E. Knox and A. Wilson)

geometrical explanations would also count as explanation by constraint. For an illustration, consider the example involving the parallel-transported stick along the spherical surface (cf. $\S 2)$. The standard geometrical explanation of $\alpha$ is independent of contingent causal, dynamical, and nomological features of the world, thereby holding with mathematical or logical necessity. (Due to this modal strength of the 'constraint', Lange would call this 'distinctly mathematical' explanation. Other explanations 'by constraint' involve grades of necessity strictly weaker than this, but nevertheless stronger than those of ordinary causal laws.) Lange argues that explanations by constraint explain by virtue of showing why the explanandum had to be the way it is, for reasons that are modally stronger than ordinary causal laws. The relative modal strength of explanatory constraints Lange in turn characterises in terms of their independence from actual causal laws.

It is undeniably true that a distinctive feature of many non-causal explanations in physics is a kind of independence or abstraction away from causal features of the world. It does not automatically follow that this feature is what makes these explanations explanatory, however. In particular, philosophers exemplifying the second trend highlighted above have argued that various purported examples of non-causal explanations work by virtue of providing dependence information, despite their extreme level of abstraction from causal features of the world.

In this spirit Jansson and Saatsi (forthcoming) critically discuss the accounts of Pincock and Lange, for example, arguing that many of the key examples motivating their accounts also involve information about non-causal dependence relations, and that this information furthermore is naturally construed as explanatory very much in the spirit of a popular counterfactual account of causal explanation (Woodward 2003). The 'minimal models' account of Batterman and Rice is critically analysed by Lange (2015), who argues that their examples can be construed as involving information about essential common features shared by otherwise heterogeneous systems. Furthermore, one could argue that these features, although highly abstract, are explanatory by virtue of showing what the explanandum depends on. (This further claim is not part of Lange's brief, however.) This would be in tune with Saatsi and Reutlinger (2018), who emphasise the role of counterfactual dependence information specifically in connection with renormalisation group explanations of critical phenomena. (See also Reutlinger 2016 and Woodward 2018.) 
For Routledge Handbook of Philosophy of Physics (Eds. E. Knox and A. Wilson)

The proponents of counterfactual accounts to explanations have sought to generalise the ideology of causal explanation to non-causal cases quite generally. This is motivated by noting that in a wide range of examples given above there is some interesting, non-trivial modal information in play that seems to inform us of the dependence of the explanandum on some other factors. For example, arguably important symmetry-based explanationsincluding those mentioned above - provide answers to counterfactual what-if-things-hadbeen-different questions (French and Saatsi 2018). Similarly, Saatsi (2018) argues that many geometrical explanations in physics — including those involving a geometrical phase - are naturally understood in counterfactual terms, and Saatsi (2017b) suggest likewise regarding explanations furnished by phase-spaces and attractors in dynamical systems theory. Bokulich (2008) appropriates the counterfactual approach to a model-based explanation of the semiclassical phenomenon of wavefunction scarring, and Reutlinger (2018) discusses various further cases in the same spirit.

Consider again, for example, the paradigmatic geometrical explanation involving a stick's parallel transport over a closed loop on a spherical surface. In Saatsi's (2018) analysis this explanation is best understood in counterfactual terms, supported by the equation that connects the shift in the stick's angle, $\alpha$, on the one hand, and the area of closed loop and the curvature of the surface, on the other. What is it that renders this explanation non-causal (or mathematical, perhaps), if the ideology of explanatory counterfactual information is apt? According to Saatsi, the difference between this non-causal geometrical explanation and causal explanations boils down to the inapplicability of the (causal) notion of intervention in the non-causal case, due to some of the explanans variables being suitably non-local. Another key feature of this kind of non-causal explanation is that the explanatory connection between the explanandum and explanans variables is too intimate to count as a contingent causal connection (Woodward 2018).

There is still a good deal of work to be done on the metaphysics and epistemology of noncausal explanations. (See Reutlinger 2017 for a recent review of philosophy of non-causal explanation, and Reutlinger and Saatsi (2018) for a collection of recent research.) The nature of purported non-causal dependence relations is murkier than their causal counterparts, for instance, and there is a prominent challenge faced by everyone in the debate: how to account for the directionality of explanation? This challenge is unsurprising in the light of the wellknown challenges to the DN-account and the subsequent appeal to causation and unification 
For Routledge Handbook of Philosophy of Physics (Eds. E. Knox and A. Wilson)

as offering a response to these challenges. Adopting the ideology of counterfactual causal accounts of explanation, sans causation, clearly leaves open the question what grounds the directionality of explanation. (For different responses, see e.g. Jansson 2015, Jansson and Saatsi forthcoming, Reutlinger 2018.) The directionality challenge has been explicitly raised against the 'minimal models' account (by Lange 2015); counterfactual accounts (by Lange 2019), and against Lange's account of distinctly mathematical explanations (by Craver and Povich 2017).

Acknowledgments. Many thanks to Alex Reutlinger and Eleanor Knox for their helpful comments on an earlier draft.

\section{References}

Batterman, R. W. (2003). 'Falling cats, parallel parking, and polarized light,' Studies in History and Philosophy of Modern Physics, 34(4), 527-557.

Batterman, R. W., \& Rice, C. C. (2014). 'Minimal model explanations,' Philosophy of Science, 81(3), 349-376.

Bokulich, A. (2008). 'Can classical structures explain quantum phenomena?' The British Journal for the Philosophy of Science, 59 (2), 217-235.

Buchel, W. (1969). 'Why is space three-dimensional?' American Journal of Physics, 37, $1222-24$.

Callender, C. (2005). 'Answers in search of a question: 'proofs' of the tri-dimensionality of space,' Studies in History and Philosophy of Modern Physics, 36(1), 113-36.

Clifton, R. (1998). 'Scientific Explanation in Quantum Theory,' unpublished manuscript. Available at unpublished manuscript. philsci-archive. pitt. edu/archive/00000091

Craver, C. F., \& Povich, M. (2017). 'The directionality of distinctively mathematical explanations,' Studies in History and Philosophy of Science, 63, 31-38. 
For Routledge Handbook of Philosophy of Physics (Eds. E. Knox and A. Wilson)

Dizadji-Bahmani, F., Frigg, R., \& Hartmann, S. (2010). 'Who’s Afraid of Nagelian Reduction,' Erkenntnis, 73(3), 393-412.

Ehrenfest, P. (1917). 'In what way does it become manifest in the fundamental laws of physics that space has three dimensions?' Proceedings of the Amsterdam Academy, 20, 200209.

French, S., \& Saatsi, J. (2018). 'Symmetries and explanatory dependencies in physics,' In Reutlinger and Saatsi (2018).

Healey, R. (2007). Gauging What's Real: The Conceptual Foundations of Contemporary Gauge Theories. New York: Oxford University Press.

Healey, R. (2017). The quantum revolution in philosophy. New York: Oxford University Press.

Hempel, C. G., \& Oppenheim, P. (1948). 'Studies in the logic of explanation,' Philosophy of Science, 15, 135-75.

Jansson, L. (2015). 'Explanatory Asymmetries: Laws of Nature Rehabilitated', Journal of Philosophy, 112(11): 577-99.

Jansson, L., \& Saatsi, J. (Forthcoming). 'Explanatory abstractions,' British Journal for the Philosophy of Science.

Lange, M. (2007). 'Laws and meta-laws of nature: conservation laws and symmetries,' Studies in History and Philosophy of Modern Physics, 38(3), 457-81.

Lange, M. (2009). 'Dimensional explanations,' Noûs, 43(4), 742-75.

Lange, M. (2015). 'On “minimal model explanations": A Reply to Batterman and Rice,' Philosophy of Science, 82(2), 292-305. 
For Routledge Handbook of Philosophy of Physics (Eds. E. Knox and A. Wilson)

Lange, M. (2017). Because without Cause: Non-causal Explanations in Science and Mathematics. Oxford: Oxford University Press.

Lange, M. (2019). 'Asymmetry as a challenge to counterfactual accounts,' Synthese, https://doi.org/10.1007/s11229-019-02317-3.

Lyre, H. (2014). 'Berry phase and quantum structure,' Studies in History and Philosophy of Modern Physics, 48, 45-51.

Morrison, M. (2015). Reconstructing Reality: Models, Mathematics, and Simulations. Oxford: Oxford University Press.

Nagel, E. (1961). The structure of science. London: Routledge and Keagan Paul.

Nerlich, G. (1979). 'What can geometry explain,' The British Journal for the Philosophy of Science, 30(1), 69-83.

Pexton, M. (2014). 'How dimensional analysis can explain,' Synthese, 191(10), 2333-51.

Pincock, C. (2007). 'A role for mathematics in the physical sciences,' Noûs, 41(2), 253-275.

Reutlinger, A. (2016). 'Is there a monist theory of causal and non-causal explanations?' Philosophy of Science, 83(5), 733-745.

Reutlinger, A. (2017). 'Explanation beyond causation? New directions in the philosophy of scientific explanation: Explanation Beyond Causation,' Philosophy Compass, 12(2), e12395.

Reutlinger, A. (2018). 'Extending the counterfactual theory of explanation,' in Reutlinger and Saatsi (2018).

Reutlinger, A. \& J. Saatsi (2018), Explanation Beyond Causation: Philosophical Perspectives on Non-Causal Explanations. Oxford: Oxford University Press. 
For Routledge Handbook of Philosophy of Physics (Eds. E. Knox and A. Wilson)

Saatsi, J. (2017a). 'Realism and the limits of explanatory reasoning,' in J. Saatsi (Ed.), The Routledge Handbook of Scientific Realism. London: Routledge.

Saatsi, J. (2017b). 'Dynamical Systems Theory and Explanatory Indispensability,' Philosophy of Science, 84(December), 1-14.

Saatsi, J. (2018). 'On Explanations from Geometry of Motion,' The British Journal for the Philosophy of Science, 69(1), 253-73.

Saatsi, J., \& Reutlinger, A. (2018). 'Taking Reductionism to the Limit: How to Rebut the Anti-Reductionist Argument from Infinite Limits,' Philosophy of Science, 85, 455-82.

Salmon, W. C. (1989). 'Four decades of scientific explanation,' in P. Kitcher \& W. C. Salmon (eds.) Minnesota Studies in the Philosophy of Science vol. 13, (pp. 3-219). Minneapolis: University of Minnesota Press.

Schaffner, K. F. (2012). 'Ernest Nagel and reduction,' The Journal of Philosophy, 109(8/9), 534-65.

Skow, B. (2014). 'Are There Non-Causal Explanations (of Particular Events),' The British Journal for the Philosophy of Science, 65(3), 445-67.

Skow, B. (2016). Reasons Why. New York: Oxford University Press.

Strevens, M. (2008). Depth: An Account of Scientific Explanation. Cambridge, Mass.: Harvard University Press.

Strevens, M. (2018). 'The mathematical route to causal understanding,' in Reutlinger and Saatsi (2018).

Sullivan, E. (Forthcoming). 'Universality Caused: The Case of Renormalization Group Explanation', European Journal for Philosophy of Science. 
For Routledge Handbook of Philosophy of Physics (Eds. E. Knox and A. Wilson)

von Bergmann, J., \& von Bergmann, H. (2007). 'Foucault pendulum through basic geometry,' American Journal of Physics, 75, 888-92.

Weatherall, J. O. (2011). 'On (some) explanations in physics,' Philosophy of Science, 78(3), 421-447.

Wilczek, F., \& Shapere, A. (1989). Geometric Phases in Physics. Singapore: World Scientific.

Woodward, J. (2003). Making Things Happen: A Causal Theory of Explanation. Oxford: Oxford University Press.

Woodward, J. (2018). 'Some varieties of non-causal explanation,' in Reutlinger and Saatsi (2018). 